\title{
Base Deficit as an Indicator of Significant Blunt Abdominal Trauma
}

Daniel Kinyuru Ojuka, Daniel Mukhwana Nyongesa, Peter Mungai Ngugi

School of Medicine, University of Nairobi

Correspondence to:Dr. Daniel Ojuka, P.O. Box 19762 - 00202, Nairobi, Kenya. Email: danielojuka@gmail.com

\begin{abstract}
Background: Blunt abdominal trauma (BAT) is an important cause of morbidity and mortality among trauma patients. Base deficit (BD) has been proposed as an early available tool alongside focused assessment with sonography for trauma (FAST) in the screening of patients suspected to have BAT and also to help guide the selective use of CT scan. Objective: To determine the use of $\mathrm{BD}$ as an indicator of significant BAT.
\end{abstract} Methods: This was an observational study carried out at the Kenyatta National Hospital from February to May 2015. Patient with suspected BAT admitted into Accident \& Emergency were enrolled. Data collected included clinical assessment, BD, FAST findings, CTscan, and laparotomy were recorded. Data was analyzed using SPSS 17.0. Comparison of mean values of BD between different groups of patients (discharged

\section{Introduction}

The prevalence of intra-abdominal injury (any injury to intraperitoneal and retroperitoneal organs including the presence of hemoperitoneum) in BAT is about $13 \%$ with $4.7 \%$ requiring therapeutic surgery or angiographic embolization to stop bleeding (1). In South Africa, the incidence of BAT is estimated to be $9.8 \%$ while a local Kenyan study puts the ratio of penetrating to BAT at $2: 1(2,3)$. Not all intra-abdominal injuries have significant clinical consequences. Most investigators define patients with significant intra-abdominal injuries as those requiring therapeutic surgery or angiographic embolization to stop hemorrhage (1).Evaluation of patients with BAT has significant diagnostic challenges even to the most experienced trauma surgeon (4).Patients with severe injuries and ongoing blood loss need immediate identification and treatment while from A\&E, managed conservatively or operated) was performed using ANOVA. Results: Patients who had significant abdominal injury had sensitivity and specificity of $82.98 \%$ and $65.91 \%$ respectively at BD of -4.15 .The PPV and NPV were $56.52 \%$ and $87.88 \%$ respectively. At this cut-off point, AUC was 0.863 $(p=0.037)$. Conclusion: Due to high NPV, BD could rule out significant abdominal injuries but can also predict need for exploratory laparotomy when less than -6.85 .

Key words: Base deficit, Blunt abdominal trauma, Predictor.

Ann Afr Surg. 2017; 14(2):61-65

DOI: http://dx.doi.org/10.4314/aas.v14i2.2

(C) 2017 Author.This work is licensed under the Creative Commons Attribution 4.0 International License.

those with seemingly less severe trauma or have unequivocal clinical signs on initial physical examination may still have significant intra-abdominal injuries, and delayed recognition may be responsible for preventable morbidity and mortality (1).The most widely used algorithms for hemodynamically unstable patients involve diagnostic modalities such as FAST or diagnostic peritoneal lavage (DPL) to determine the need for urgent surgical intervention (5). Studies have shown that $\mathrm{BD}$ is a laboratory investigation that can aid in screening patients with suspected BAT alongside FAST (6-8). Some authors have emphasized the importance of obtaining it early during the evaluation of blunt traumapatients as it can reliably predict not just ongoing hemorrhage but significant intraabdominalinjury and the need for surgical intervention (6). In most studies a BD of $-6 \mathrm{mEq} / \mathrm{L}$ or less has been 
shown to be predictive of significant intra-abdominal injury in BAT (1). Abdominal $\mathrm{CT}$ is the most commonly used modality for diagnostic evaluation of patients with BAT who are hemodynamically stable. However, less than $20 \%$ of abdominal CT scans obtained in these patients are positive for intraabdominal injury and less than $3 \%$ have injuries that require surgical intervention or angiographic embolization (5). Deunk et al. proposed a selective criterion in victims of blunt trauma, based on clinical, radiological, laboratory and ultrasound exam to guide use of CT scan (9). Among the laboratory investigations, he proposed a $\mathrm{BD}$ of $<-3 \mathrm{mEq} / 1$ as predictive of the need for evaluation by CT scan (9). However, there is still paucity of data on the usefulness of BD in BAT. There is no such study in our local set up. This study seeks to validate the usefulness of BD in BAT as an indicator of significant intra-abdominal injury.

\section{Methods}

This was a prospective observational study carried out in the accident and Emergency (A\&E) and the surgical Wards at Kenyatta National Hospital (KNH) from February 2015 to May 2015. We included patients who were $12 \mathrm{yrs}$ old and above, admitted to A\&E and the surgical wards and had suspected BAT. Informed consent was sought from the recruited patients or their next of kin. We excluded patients who were below $12 \mathrm{yrs}$ of age or patients who had been investigated and resuscitated elsewhere before arrival or patients with extra-abdominal injuries.Demographic information (age, sex) was collected for enrolled patients as well as medical histories and clinical data: initial GCS, blood pressure, pulse rate,temperature, presence or absence of peritonitis and presence or absence of extra-abdominal injuries. Clinical evaluation and decision on who had BAT was by the attending resident in the admitting firms. Arterial blood samples $(0.5 \mathrm{mls}$ in heparinized $2.5 \mathrm{ml}$ syringes) was taken for all patients suspected to have BAT on admission to A\&E. These samples were sent immediately for blood gas analysis using the blood gas analyzer (Siemens Rapid Lab 348) in the Intensive Care Unit (ICU) laboratory at KNH. All patients suspected to have BAT underwent screening by FAST. Abdominal CT Scan was obtained for patients who had unequivocal findings and remained clinically stable.
Clinical, radiological and laboratory data as well as surgical results in operated patients were recorded in the data sheet. Significant abdominal injury was defined as any injury requiring surgical intervention.Data was entered into SPSS version 17 for analysis. Comparison of mean values of BD between significant abdominal injury and non- significant abdominal injury were performed. Sensitivity, specificity, NPV, PPV and likelihood ratio was calculated by correlating $\mathrm{BD}$ and the findings on FAST, CT scan and intra-operatively. All results were reported with $95 \%$ confidence interval. Probabilities ( $p$ value) of less than 0.05 were considered statistically significant. This study was approved by the Institutional Review Committee.

\section{Results}

A total of 134 patients with suspected BAT were recruited during the study period. Two patients were excluded from the study because one died while awaiting explorative laparotomy while the other was discharged from A\&E did notanswer calls when contacted. Out of the 132 analyzed, 108 patients $(81.82 \%)$ were males while $24(18.18 \%)$ patients were females giving a male to female ratio of 4.9:1. Eightysix patients were discharged from (A\&E), 7 patients were discharged from the wards in $<48$ hours after ruling out abdominal injury, 33 patients were managed conservatively for abdominal injury while 13 patients underwent exploratory laparotomy (Table 1).

Table 1: Patients Outcome and its Correlation with BD
\begin{tabular}{|l|l|l|}
\hline Outcome & BD of $<-6$ & BD of $>\mathbf{6}$ \\
\hline Discharged from A\&E & 3 & 83 \\
\hline Managed conservatively & 25 & 8 \\
\hline Operated & 12 & 1 \\
\hline
\end{tabular}

A\&E - Accident and Emergency; BD - base deficit

FAST was performed on all the recruited patients. Thirty-eight patients $(28.79 \%)$ had a positive FAST while $84(63.64 \%)$ had a negative FAST. Eight patients had a negative FAST despite having abdominal injuries (AI). Thirty-eight patients underwent CT scan, out of which $8(21 \%)$ had no injuries while only $3(7 \%)$ patients had significant injuries requiring surgical intervention. One patient $(2.6 \%)$ had a normal CT scan result despite having significant abdominal injury upon laparotomy. Forty-six (34.85\%) patients were confirmed to have AI by FAST, CT scan, laparotomy or a combination of methods while the rest, 86(65.15\%) 
did not have abdominal injury. Patients who had abdominal injury versus those who did not, the cut-off value at which the greatest sum of sensitivity $82.98 \%$ $(69.19 \%$ to $92.35 \%)$ and specificity $65.91 \%(55.03 \%$ to $75.68 \%$ ) was obtained for base deficit was -4.15 . The PPV was $56.52 \%(44.04 \%$ to $68.42 \%)$ and the NPV was $87.88 \%$ (77.51\% to $94.62 \%)$. The LR $(+)$ was 2.43 (1.77 to 3.35) while the LR (-) was 0.26 (0.13 to 0.49$)$ at this cut-off point, AUC $(95 \% \mathrm{CI})$ for BD was 0.863 $(\mathrm{P}=0.037)$ (Table 2, Figure 1).

Table 2: Predictive Values for Abdominal Injury Versus Non Abdominal Injury

\begin{tabular}{|l|l|l|}
\hline Statistic & Value & $\mathbf{9 5 \%}$ CI \\
\hline Sensitivity & $82.98 \%$ & $69.19 \%$ to $92.35 \%$ \\
\hline Specificity & $65.91 \%$ & $55.03 \%$ to $75.68 \%$ \\
\hline Positive Likelihood Ratio & 2.43 & 1.77 to 3.35 \\
\hline Negative Likelihood Ratio & 0.26 & 0.13 to 0.49 \\
\hline Positive Predictive Value & $56.52 \%$ & $44.04 \%$ to $68.42 \%$ \\
\hline Negative Predictive Value & $87.88 \%$ & $77.51 \%$ to $94.62 \%$ \\
\hline
\end{tabular}

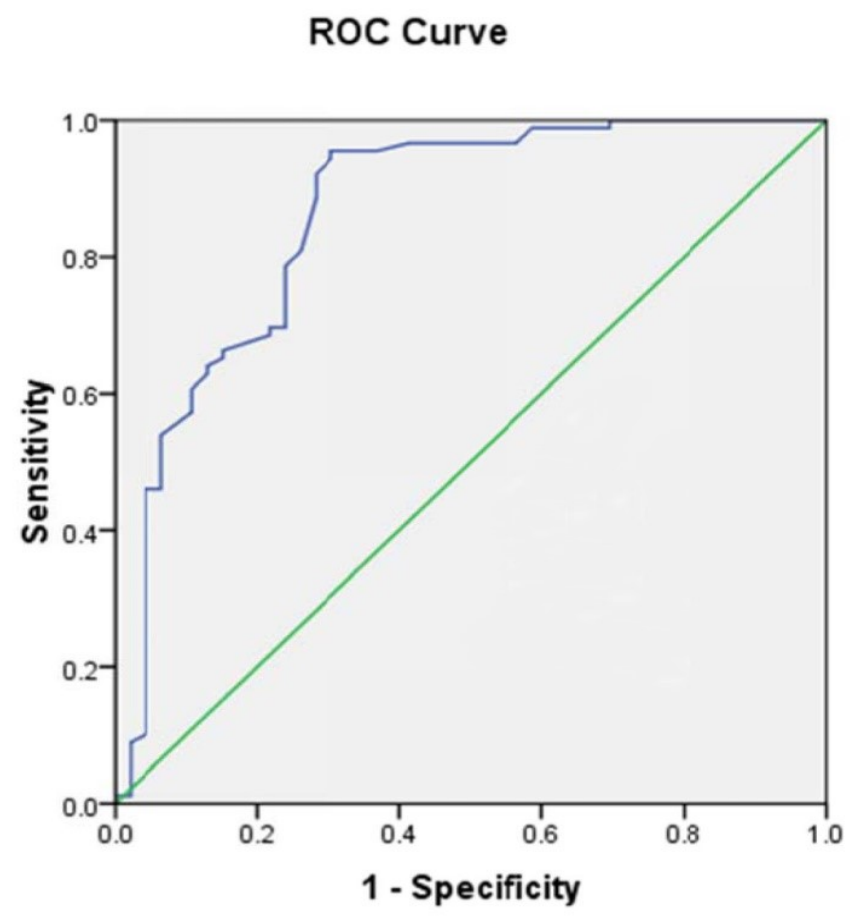

Diagonal segments are produced by ties

Figure 1: ROC curve for BD versus abdominal injury

Predictive values for BD versus the need for operative management. Patients who underwent explorative laparotomy versus those who did not, the cutoff value at which the greatest sum of sensitivity $73.33 \%(44.90 \%$ to $92.21 \%$ ) and specificity $58.06 \%$ ( $39.08 \%$ to $75.45 \%)$ was obtained for base deficit was -4.15 . The PPV was $45.83 \%(25.55 \%$ to $67.18 \%)$ and the NPV was $81.82 \%(59.72 \%$ to $94.81 \%)$. The LR $(+)$ was $1.75 \mathrm{~b}$ (1.05 to 2.93) while the LR (-) was 0.46 (0.19 to 1.12 ) at this cut-off point, AUC $(95 \% \mathrm{CI})$ for BD was 0.863 $(\mathrm{P}=0.037)$ (Table 3, Figure 2).

Table 3: Predictive Values of BD for the Likelihood of Laparotomy

\begin{tabular}{l|l|l|l|}
\hline Statistic & Value & $\mathbf{9 5 \%}$ CI \\
\hline Sensitivity & $73.33 \%$ & $44.90 \%$ to $92.21 \%$ \\
\hline Specificity & $58.06 \%$ & $39.08 \%$ to $75.45 \%$ \\
\hline Positive Likelihood Ratio & 1.75 & 1.05 to 2.93 \\
\hline Negative Likelihood Ratio & 0.46 & 0.19 to 1.12 \\
\hline Disease prevalence & $32.61 \%$ & $19.53 \%$ to $48.02 \%$ \\
\hline Positive Predictive Value & $45.83 \%$ & $25.55 \%$ to $67.18 \%$ \\
\hline Negative Predictive Value & $81.82 \%$ & $59.72 \%$ to $94.81 \%$ \\
\hline
\end{tabular}

ROC Curve

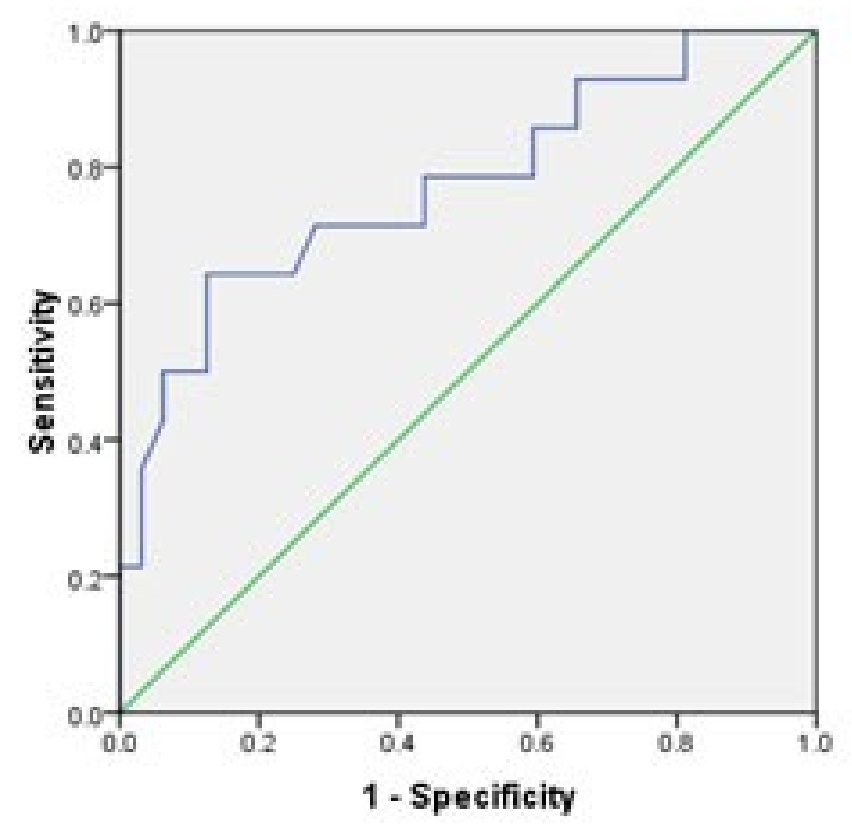

Diagonal segments are produced by ties

Figure 2: ROC for BD versus likely hood of undergoing exploratory laparotomy

\section{Discussion}

This study was conducted to determine the usefulness of base deficit in the diagnosis of significant abdominal injuries in adults who suffered suspected blunt abdominal trauma at Kenyatta National Hospital. The results of the study show that BAT is a predominantly male problem, affecting them 4.6 times more than the females. The mean (SD) age of the patients was 
$33.2 \pm 15.1$. These findings are consistent with studies in many centers around the world which show that trauma largely affects males who are in the first 4 decades of their life (1). Musau et al. made a similar observation in a study conducted at this same institution (3).

Regarding BD and its usefulness in the diagnosis of BAT, the results showed that BD less than or equal to 4.15 can determine with high accuracy intra-abdominal injury while a BD less than or equal to -6.8 predicts quite accurately the need for surgical intervention among persons aged 12 years and above. In the current study, we observed that among the 46 patients who had abdominal injuries, 39 patients had a BD of $<-4.15$ while out of the 13 patients who underwent explorative laparotomy 12 had a BD of less than -6.85 . Of the 86 patients who were confirmed not to have abdominal injury, only 3 patients had a $\mathrm{BD}<-6.85$. These findings compare well with Hojjat et al. findings regarding BD and its accuracy in the diagnosis of BAT (10). They obtained a cut point for the amount of BD at -4. 55 . Their study also showed that out of 39 patients who had sustained blunt abdominal trauma, 34 patients had a BD $<-4.15(10)$. A significant difference between their study and our study is that it was done on children under the age of 12 years (10). In a similar study to ours though with a bigger sample size of 400 patients, Mofidi et al. established that a BD greater than -6 showed lack of intra-abdominal injury while a BD less than or equal to -6 strongly indicated presence of intraabdominal injury and bleeding (11). Patients with a BD of -6 or lower achieved more laparotomy and blood transfusion compared with patients with a BD more than -6 . He showed that $68 \%$ of patients with a BD of less than -6 required transfusion (12). These findings are almost similar to our findings regarding presence of significant intra-abdominal injury in BAT. In another study a BD less than or equal to -6 was the single most significant indicator of AI ( $\mathrm{P}$ less than or equal to

.0001), and the odds ratio for AI increased with each category of increasing severity of BD (12).

The only study that is at a slight variance with our findings is by Deunk et al. They identified among other factors a base deficit lower than $-3 \mathrm{mEq} / \mathrm{L}$ in arterial blood gases as an independent predictor of significant intra-abdominal injury (9). Our study established that the sensitivity and specificity of BD as a diagnostic tool for significant BAT was $82.98 \%(69.1 \%$ to $92.35 \%)$ and $65.91 \%$ (55.0\% to $75.68 \%)$ respectively. These figures vary slightly from figures established by other studies. In fact, there are no studies that showed similar results of sensitivity and specificity. Hojjat et al showed sensitivity of $91 \%$ and specificity of $86 \%$ while Mofidi et al. established a sensitivity and a specificity of $88.2 \%$ and $95.2 \%$ respectively $(10,11)$. Despite the differences, all the studies showed that $\mathrm{BD}$ is both sensitive and specific in determining presence of significant intraabdominal injury in BAT. Similarly, different studies showed variable results of PPV and NPV. The PPV and NPV established by our study were $56.52 \%$ and $88.7 \%$ respectively. According to Hojjat et al. the PPV and the NPV was $71.05 \%$ and $97.7 \%$ respectively. Mofidi et al. found a PPV and a NPV of $79 \%$ and $97.5 \%$ respectively $(10,11)$.

Thirty-eight patients underwent CT scanning out of which $8(21 \%)$ had injuries while only $3(7 \%)$ patients had significant injuries requiring surgical intervention. These results are consistent with findings by Deunk et al. They demonstrated that just about $20 \%$ of patients suspected to have BAT will have injuries on CT scan while less than $3 \%$ will have serious injuries requiring therapeutic intervention (9). In most studies a BD of -6 $\mathrm{mEq} / \mathrm{L}$ or less has been shown to be predictive of significant intra-abdominal injury in BAT (1). In our study, all patients except 1 who had significant findings requiring exploratory laparotomy had a $\mathrm{BD}$ of less than $-6.85 \mathrm{MEq} / \mathrm{L}$.

The study was hospital-based, and was depended on the number of patients attended to at $\mathrm{KNH}$ within the study period. It was therefore not representative of the true picture of the entire Kenyan population.

\section{Conclusion}

The findings of our study show that BD is an early available tool that can be used to predict presence of AI as well as significant AI (injuries requiring exploratory laparotomy). At a cut-off of -4.15 , the likelihood of abdominal injury is very high that an objective evaluation using imaging is warranted. On the otherhand, a normal BD, though an important indicator of absence of injury does not rule out presence of injury, however our findings show that a patient with significant intra-abdominal injuries requiring surgical intervention is unlikely to have a $\mathrm{BD}>-6.85 \mathrm{MEq} / \mathrm{L}$. 


\section{References}

1. Nishijima DK, Simel DL, Wisner DH, et al. Does This Adult Patient Have a Blunt Intra-Abdominal Injury? JAMA.2012; 307(14):1517-27

2. Mnguni MN, Muckart DJ, Madiba TE. Abdominal Trauma in Durban, South Africa: Factors Influencing Outcome. Int Surg. 2012; 97(2): 161-8

3. Musau P, Jani PG, Owillah FA. Pattern and Outcome of Abdominal Injuries at Kenyatta National Hospital, Nairobi. East Afr Med J. 2006; 83(1):37-43

4. Farrath S, Parreira JG, Olliari CB, et al. Identifying Severe Abdominal Injuries during the Initial Assessment in Blunt Trauma Patients. Rev Col Bras Cir. 2013; 40(4): 305-11

5. Dunham CM, Sipe EK, Peluso L. Emergency Department Spirometric Volume and Base Deficit Delineate Risk for Torso Injury in Stable Patients. BMC Surg. 2004; 4:3

6. Sabogal CE, Rivera AF, Higuera J, et al. Lactate and Base Deficit in Trauma: Prognostic Value. Rev Colomb Anesthesiol. 2014;42(1):60-4

7. Chawla LS, Nader A, Nelson T, et al. Utilization of Base Deficit and Reliability of Base Deficit as a Surrogate for Serum Lactate in the Peri-operative. BMC Anesthesiol. 2010; 10:16doi: 10.1186/14712253-10-16.

8. Husain FA, Martin MJ, Mullenix PS, et al. Serum Lactate and Base Deficit as Predictors of Mortality and Morbidity. Am J Surg. 2003; 185(5):485-91.

9. Deunk J, Brink M, Dekker H, et al. Predictors for the Selection of Patients for Abdominal CT after Blunt Trauma: A Proposal for a Diagnostic Algorithm. Ann of Surg. 2010; 251(3) ;512-20

10. Hojjat D, Mojtaba S, Majid S, et al. Accuracy of Base Deficit in Diagnosis of Intra-Abdominal Injury in Pediatrics with Blunt Abdominal Trauma. J. Basic. Appl. Sci. Res.2013; 3(6); 511-5.

11. Mofidi M, Hasani A, Kianmehr N. Determining the Accuracy of Base Deficit in Diagnosis of Intraabdominal Injury in Patients with Blunt Abdominal Trauma. Am J Emerg Med. 2010;28(8):933-6

12. Davis JW, Mackersie RC, Holbrook TL, et al. Base Deficit as an Indicator of Significant Abdominal Injury. Ann Emerg Med. 1991;20(8):842-7 